\title{
Guest Editorial for the Special Issue on Unmanned Autonomous Vehicles
}

\author{
Sesh Commuri · Rafael Fierro
}

Published online: 27 June 2009

(C) Springer Science + Business Media B.V. 2009

\section{Dear Colleagues,}

It is a great privilege to bring to you two special issues in Volume 56, covering the state-of-the art research in the area of Autonomous Vehicles. The increased interest in this area during the past decade has led to the development of several unmanned systems for applications from surveillance to space exploration to environmental monitoring. The two issues highlight the recent developments in this exciting area and the challenges to the realization of high-confidence unmanned systems for realworld operations.

The coordination and planning of autonomous vehicles are presented in Issue 1. Zou and Pagilla demonstrate the control of multiple mobile robots using distributed constraint force. Moore and Passino develop a rigorous algorithm to enable a group of autonomous vehicles to effectively patrol an environment significantly larger than their communication and sensing radii. Derenick, Spletzer, and Hsieh provide an optimal approach to guaranteed performance during collaborative target tracking. Path planning using Information Roadmaps is presented by Zhang, Ferrari, and Qian. Schuresko and Cortés explore the motion constraints for algebraic connectivity in mobile robot teams.

The control of autonomous vehicles is addressed in Issue 2. Adaptive backstepping approach to handle nonlinear aspects of the aircraft dynamics (Das, Lewis, and Subbarao), neural networks based control of robot formations (Dierks and Jagannathan), and control of systems with rigidly mounted sensors (Kim, Zhang,

S. Commuri $(\bowtie)$

School of Electrical \& Computer Engineering, University of Oklahoma,

Norman, OK, USA

e-mail: scommuri@ou.edu

R. Fierro $(\bowtie)$

Department of Electrical \& Computer Engineering, University of New Mexico,

Albuquerque, NM, USA

e-mail: rfierro@ece.unm.edu 
and Egerstedt) demonstrate how performance can be guaranteed in the face of unknown nonlinear behavior characteristics of unmanned autonomous vehicles. Communication issues arising in the control of networked systems are addressed in the paper by Frew, Lawrence, Elston, Gray and Argrow. Communication-aware motion planning of mobile sensor nodes is presented by Mostofi.

While it has been a challenge to pull together such diverse topics into a special issue, these two issues stand out for their technical content and rigor. The papers demonstrate the technical challenges and the importance of these areas to the development of Autonomous Vehicle Systems.

We hope you enjoy these two issues. 